\title{
Project Scheduling: Survey and Research Potentials
}

\author{
Asmaa Atef \\ Department of Operations \\ Research, Faculty of \\ Computers and Informatics, \\ Zagazig University, El-Zera \\ Square, Zagazig, Sharqiyah, \\ Egypt
}

\author{
Mohamed Abdel-Baset \\ Department of Operations \\ Research, Faculty of \\ Computers and Informatics, \\ Zagazig University, El-Zera \\ Square, Zagazig, Sharqiyah, \\ Egypt
}

\author{
Ibrahim El-henawy \\ Department of Computer \\ Science, Faculty of Computers \\ and Informatics, Zagazig \\ University, El-Zera Square, \\ Zagazig, Sharqiyah, Egypt.
}

\begin{abstract}
Resource constrained project scheduling problem (RCPSP) consists of activities that must be scheduled based on dependencies relationships and priorities of activities. In the recent years there have been many survey papers around the area of project scheduling, as many researchers developed both exact and heuristic scheduling schemes. This paper give an over view around the resource constrained project scheduling problem (RCPSP).
\end{abstract}

Keywords: Resource allocation; constrained resource scheduling; scheduling techniques; Project management

\section{INTRODUCTION}

Scheduling is the way we actually manage a project. Without scheduling, nothing or nobody is managing the project and hence amounts to failure of a project. Scheduling describes guidance and pathway for a project to run. It defines certain milestones and deliverables which need to be achieved on a timely basis for successful completion of a project. Monitoring the schedule provides an idea of the impact the current problems are having on the project, and provides opportunities to enhance or reduce the scope of a milestone/phase in the project. It also provides a medium for continuous feedback on how the project is progressing and if there are issues that need to be dealt with or if the client needs to be told about a delay in delivery (et al Shruti Gauri).

To organize and complete your projects in a timely, quality and financially responsible manner, you need to schedule projects carefully. Effective project scheduling plays a crucial role in ensuring project success. To keep projects on track, set realistic time frames, assign resources appropriately and manage quality to decrease product errors. This typically results in reduced costs and increased customer satisfaction. Important factors include financial, documentation, management and quality assurance.

Project scheduling impacts the overall finances of a project. Time constraints require project managers to schedule resources effectively. This is particularly true when resources must have highly specialized skills and knowledge in order to complete a task or when costly materials are required. Completing a project in a short time frame typically costs more because additional resources or expedited materials are needed. With accurate project scheduling, realistic estimates and accurate projections prevent last-minute orders that drive up costs.

A well-managed project will require a proper schedule and related documents. Project schedule will help to organize all the related tasks related to project in a easy to manage way. Project managers can easily see and manage tasks. Without a schedule you will never know when you're going to finish your project. Some people may just start projects without a proper schedule. If you don't make a project schedule, it is very likely that you may have to spend sleepless nights rushing to finish the project on time. And at the end of the day, the quality of the finished project might be poor.

Estimating the duration and making a schedule is not a thing which is possible for the inexperienced. You will need some amount of experience before you will be able to estimate. If you have never worked on a software project or never implemented an application, you won't be able to draw a time-line very well. Project scheduling ensures one task gets completed in a quality manner before the next task in the process begins. By assuring that quality measures meet expectations at every step of the way, you ensure that managers and team members address problems as they arise and don't wait until the end.

No major issues should appear upon completion because you've established quality controls from the very beginning of the scheduling process. Effective project managers understand that ensuring quality control involves managing risks and exploiting opportunities to speed up the schedule when possible 
to beat the competition and achieve or maintain a competitive edge with a more reliable product.

\subsection{The Resource-Constrained Project Scheduling Problem}

A combinatorial optimization problem informally, a resource-constrained project scheduling problem (RCPSP) considers resources of limited availability and activities of known durations and resource requests, linked by precedence relations. The problem consists of finding a schedule of minimal duration by assigning a start time to each activity such that the precedence relations and the resource availabilities are respected.

\subsection{Project management and scheduling}

Project management and scheduling are major issues for every company or organization facing rapid changes in its environment. Furthermore, project scheduling techniques have become indispensable for the attainment of effective-ness and efficiency of processes. This special issue collects 11 carefully selected papers which deal with optimization or decision analysis problems in the field of project management and scheduling. It covers a considerable range of topics, including solution methods for classical project scheduling problems (Bianco and Caramia, Kone' et al., Lim et al., Van Peteghem and Vanhoucke), mixed-integer programming (MIP) formulations in order to solve scheduling problems with commercial MIP-solvers (Bianco and Caramia, Kone et al.), models and solution algorithms for multi-project scheduling (Besikci et al.), extensions of the classical resourceconstrained project scheduling problem (Hartmann), models and solution approaches integrating decisions at different managerial levels such as selection and scheduling, or scheduling and control (Gutjahr and Froeschl, Hazir and Schmidt), risk in project management and scheduling (Tian and Demeulemeester, Artigues et al., Hazir and Schmidt, Gutjahr and Froeschl), and the assignment of resources to different stakeholders (Fink and Homberger, Besikci et al.).

The operations research techniques employed include common approaches such as metaheuristics (Lim et al., Fink and Homberger, Van Peteghem and Vanhoucke, Besikci et al., Gutjah and Froeschl) and mixed-integer programming (Bianco and Caramia, Kone et al.), but also specific approaches such as scenario-relaxation algorithms (Artigues et al.), a Lagrangian-based algorithm (Beşikci et al.), and a Frank-Wolfe type algorithm (Gutjahr and Froeschl). This special issue thus covers a broad range of models, algorithms and applications. It demonstrates that 55 years after the invention of CPM and PERT, the field is more innovative and lively than ever. We encourage the readers to seek inspiration from this unique collection of papers and to continue work in this area, for instance by applying these results in different application areas, by extending these models further, or by developing even better algorithms for solving them efficiently.

\section{LITERATURE REVIEW}

The project scheduling problems of small sizes can be solved by the traditional optimization techniques. However, as the number of projects and size of the project in terms of number of activities increase, the problem becomes more complex .Further, the complexity increases when variety of resources is considered. In this context, it is not feasible to develop the projects' schedules by using the traditional optimization techniques. The benefit of traditional optimization techniques cannot be utilized for generating the schedule of multiple projects simultaneously. Accordingly, researchers have developed several heuristic algorithm and rules and Meta heuristic methods for multiple resource scheduling and multi- project scheduling. Scheduling and allocation of resources for multiple projects is a non-polynomial (NP) hard problem and more difficult than a single project. Traditional optimization methods have been used in the literature to solve the multi project scheduling problems. The categorization of solution procedures for scheduling problem are classified as cited in Shouman et al [4,5], Rainer Kolisch and Sonke Hartmann [6] into priority rule-based-X-pass methods, classical metaheuristics, non-standard meta-heuristics, and other heuristics. In X-pass methods a priority order is given for each specified activity included in the project under either a serial and/or parallel scheduling generated strategy. X-pass methods have been summarized in Alvaerz-Valdes and Tamarit [7], Boctor [8], Cooper [9,10], Davies [11], Davies Atterson [12], Elsayed [13], Klein [14], Kolisch [15,16,17], Kolisch and Drexl [18,19] Lawrence [20], Li and Willis [21], Ozdamar and Ulusoy [22,23,243], Patterson [25,26], Schirmer [27], 
Schirmer and Riesenberg [28], Thesen [29], Thomas and Salhi [30], Ulusoy and Ozdamar [31], Valls et al. [32], and Whitehouse and Brown [33]. Shouman et al $[4,5]$ proposed fifty-five heuristic rules for scheduling projects. These heuristic rules are tested for single and multiple resource(s) constrained projects using fifty test problems. The projects are classified into different categories. The performances of the scheduling process using these heuristic rules for the considered project categories are discussed and evaluated. The performance of these scheduling rules are evaluated and appeared promising tendencies in scheduling process for single and multiple oriented critical resources(s). Also he proposed a genetic algorithm for scheduling the same test instances and compared the resulted schedules by those rendered from heuristic rules and proofs the robustness of heuristic rules performance. Lova et al [34] presents some heuristics based on priority rule for the MRCPSP with renewable resources are analyzed. These methods are very important in the construction of more sophisticated heuristics as random sampling techniques or meta-heuristics. Hence, additional efforts in order to obtain better heuristics based on priority rule are justified. Three components of this type of heuristics are analyzed: scheduling generation scheme, priority rule and mode selection rule. Based on a well-known set of 240 randomly generated project instances, it is shown that the single-pass and multi-pass heuristics based on priority rules developed in this work greatly outperforms the ones previously published. The serial schedule generation scheme greatly outperforms the parallel scheme with the majority of the priority rules tested justifying the higher computational effort required by the former. Finally, a multi-pass method that combines eight heuristics based on priority rule obtains the lowest average deviation with respect to the critical path length $(32.0 \%)$, thus being the best deterministic heuristic for this problem. Myszkowski et al [35] presents some novel scheduling heuristics for Multi-Skill Resource-Constrained Project Scheduling Problem and compared to state-of-the-art priority rules, based on task duration, resource salaries and precedence relations. New heuristics stand an aggregation of known methods, but are enhanced by skills domain. The goal of the paper is to investigate, whether evaluated methods can be used as robustness enhancement tools in meta-heuristics, mostly evolutionary algorithms. Experiments have been performed using artificially created dataset instances, based on real-world instances. Obtained results prove that such methods stand interesting feature that can be included to more complex methods and increase their robustness. Colak et al [36] consider the multi-mode resource-constrained project scheduling problem (MRCPSP) with renewable resources. In MRCPSP, an activity can be executed in one of many possible modes; each mode having different resource requirements and accordingly different activity durations. We assume that all resources are renewable from period to period, such as labor and machines. A solution to this problem basically involves two decisions - (i) The start time for each activity and (ii) the mode for each activity. Given the NP-Hard nature of the problem, heuristics and meta-heuristics are used to solve larger instances of this problem. A heuristic for this type of problem involves a combination of two priority rules - one for each of the two decisions. Heuristics generally tend to be greedy in nature. In this study we propose two non-greedy heuristics for mode selection which perform better than greedy heuristics. In addition, we study the effect of double justification and backward/forward scheduling for the MRCPS. We also study the effect of serial vs. parallel scheduling. We found that all these elements improved the solution quality. Finally an adaptive meta-heuristic procedure based on neural networks is proposed which further improves the solution quality. The effectiveness of these proposed approaches, compared to existing approaches, is demonstrated through empirical testing on two well known sets of benchmark problems. Chen and Lo [37] present and evaluate a modified ant colony optimization (ACO) approach for the resource-constrained project scheduling problems. A modified ant colony system is proposed to solve the resource-constrained scheduling problems. A two-dimensional matrix is proposed in this study for scheduling activities with time, and it has a parallel scheme for solving project scheduling problems. There are two designed heuristic is proposed. The dynamic rule is designed to modify the latest starting time of activities and hence the heuristic function. In exploration of the search solution space, this investigation proposes a delay solution generation rule to escape the local optimal solution. Simulation results demonstrate that the proposed modified ant colony system algorithm 
provides an effective and efficient approach for solving project scheduling problems with resource constraints. Bastani and Yakhchali [38] proposed OR model for multi-mode resource-constrained project scheduling problem (MRCPSP), in which multiple execution modes are available for each of the activities of the project and pre-emptive extension of the activities which allows activity splitting is introduced. Also two important constraints about the length of the split and the number of split in each activity are added. In this paper each activity split up to $\mathrm{N}$ division is allowed and the length of each activity up to $\mathrm{X}$ is limited. Setup time for each pre-emption, is considered and setup time to objective function is added. This model is compared to other models that have been presented until now and the advantages of this model in comparison with others are explained. At last the most useful methods to solve this model is presented. A new recombination operator is presented by Andreica and Chira [39] for permutation based encoding has been proposed in this paper. This operator is suitable to Resource-Constrained Project Scheduling Problem and Multi-Mode ResourceConstrained Project Scheduling Problem because it preserves the precedence constraints when obtaining the offspring from feasible parents. The main feature of the proposed operator is the use of genetic information from the best individual besides the two parents considered for recombination. Experimental results performed on ProGen project instances indicate a superior performance of the proposed operator, thus emphasizing the role that recombination has in accelerating the search in an evolutionary process. An extensive study of all recombination operators used for RCPSP and MRCPSP will be performed and the experiments will be extended to more instances with more activities. Buddhaklsomsiri and Kim [40] introduced a priority rule-based heuristic for the multi-mode resourceconstrained project scheduling problem with the splitting of activities around unavailable resources allowed. All resources considered are renewable and each resource unit may not be available at all times due to resource vacations, which are known in advance. A new concept called moving resource strength is developed to help identify project situations where activity splitting is likely to be beneficial during scheduling. The moving resource strength concept is implemented in priority rule- based heuristics to control activity splitting when scheduling. Multiple comparisons of the performance of combination of activity-mode priority rules used in the heuristics are provided. Computational experiments demonstrate the effectiveness of the heuristic in reducing project makespan, and minimizing activity splitting. Browning and Yasine [41] address the static resource-constrained multiproject scheduling problem (RCMPSP) with two lateness objectives, project lateness and portfolio lateness. In this context, past research has reported conflicting results on the performance of activity priority rule heuristics and does not provide managers with clear guidance on which rule to use in various situations. Using recently improved measures for RCMPSP characteristics, they conducted a comprehensive analysis of 20 priority rules on 12,320 test problems generated to the specifications of project-, activity-, and resource-related characteristics-including network complexity and resource distribution and contention. They found several situations in which widely advocated priority rules perform poorly and confirmed that portfolio managers and project managers will prefer different priority rules depending on their local or global objectives. The results are presented in two decision tables, the practical use of which requires managers to do only a rough, qualitative characterization of their projects in terms of complexity, degree of resource contention, and resource distribution. $\mathrm{He}$ and Zhang [42] proposed a dynamic priority rulebased forward-backward heuristic algorithm (FBHA). The FBHA optimizes resource allocation by shifting non-critical activities within their forward free float (FFF), forward total float (FTF) and backward free float (BFF), successively. A project is divided into several phases during each forward/backward scheduling module. In each phase, the shifting sequence and days of non-critical activities depend on a dynamic priority rule set. The FBHA is integrated into the Microsoft Project 2007 commercial software package to improve the performance of the software and facilitate the project planners. Singh [43] introduces an attempt to integrate the project priorities with the project schedule development. A hybrid algorithm was developed to accomplish this task. The presented algorithm is a new method for generating the schedule of any multi-project resource constrained scheduling problem where, each project has a 
defined criticality. The proposed method was validated with a case study under various scenarios. Experimental results were compared with existing priority dispatching rules. Experimental results showed the superiority of the proposed method with the existing priority dispatching rules under different operating conditions. In real project management environment, a penalty is imposed if a project completes after its due date. Some projects carry higher penalty than others. In this context, project manager can make a tradeoff among the projects penalties and can develop the cost effective project schedule, which satisfies the customer requirements. In this context, the proposed algorithm would be beneficial for project manager's to deal with these conditions. In the future, research could explore the possibility of integrating other knowledge areas including risk management and procurement management with the project schedule development. Lova and Tormos [44] presents an algorithm to study the effect of the schedule generation schemes - serial or parallel - and priority rules - MINLFT, MINSLK, MAXTWK, SASP or FCFS - with two approaches multi-project and single-project. The time criteria considered are the mean project delay and the multiproject duration increase. Through an extensive computational study, results show that with the parallel scheduling generation scheme and the multiproject approach the project manager can obtain a good multi-project schedule with the time criterion selected: minimizing mean project delay or minimizing multi-project duration increase. New heuristics - based on priority rules with a two-phase approach - that outperform classical ones are proposed to minimize mean project delay with a multi-project approach. Finally, the best heuristics analyses are evaluated together with a representative sample of commercial project management software. Browning and Yassine [45] introduce the static resource-constrained multi-project scheduling problem (RCMPSP)with two lateness objectives,projectlatenessandportfoliolateness.Inthisc ontext,pastresearchhasreported conflicting results on the performance of activity priority rule heuristics and does not provide managers with clear guidance on which rule to use in various situations. Using recently improved measures for RCMPSP characteristics, they conducted a comprehensive analysis of 20 priority rules on 12,320 test problems generated to the specifications of project-, activity-, and resource-related characteristics including network complexity and resource distribution and contention. We found several situations in which widely advocated priority rules perform poorly. They also confirmed that portfolio managers and project managers will prefer different priority rules depending on their local or global objectives.

\section{CONCLUSION AND FUTURE WORK}

Project scheduling is very important topic in project management. In this survey we have attempted to review the resource constrained project scheduling problem. We give emphasis to the heuristic rules used as inputs to the scheduling process. No one rule can achieve the best schedules in all projects as each project has special characteristics. So we develop system of large number of heuristic rules for scheduling multiple resource constrained projects to adapt to the variation of projects [46].

We encourage researchers to develop flexible heuristic decision-making procedures to meet the needs of practitioners. Also encourage researchers to develop genetic systems in scheduling based on heuristic rules that can make results of scheduling are better than genetic system from scratch.

Develop heuristic procedures in multi-mode resource constrained projects scheduling problems [46].

\section{REFERENCES}

[1] A., A., Najafi, and F., Azimi, "A Priority RuleBased Heuristic for Resource Investment Project Scheduling Problem with Discounted Cash Flows and Tardiness Penalties", Mathematical Problems in EngineeringVolume 2009 (2009), Article ID 106425, 10 pages doi:10.1155/2009/106425.

[2] J., M., Nicholas, and H., Steyn, "Project Management for Business, Engineering, and Technology "Business \& Economics press, 2088.

[3] M.,Vanhoucke, "Optimizing Regular Scheduling Objectives: Priority Rule Based Scheduling," $P M$ Knowledge Center, 2012.

[4] M., A. Shouman M.S. Ibrahim M. Khater A.A Elfrgany "Some Heuristic Rules for Scheduling Single and Multiple Resources Constrained Projects" , AEJ, Journal , Alex. 2007.

[5] M.A., Shouman, A.Z., Ghafagy, M.A., Zaghloul, and A., Bou-Shaala, "New Heuristics for Scheduling Single Constrained Resource Projects", AEJ, Journal, Vol. 38, No. 3, 1999.

[6] R., Kolisch, and S., Hartmann, "Experimental Investigation of Heuristics for Resource-constrained 
Project Scheduling: Update". European Journal of Operational Research, 2005

[7] R., Alvarez-Valdes, and J.M., Tamarit, "Heuristic Algorithms for Resource-constrained Project Scheduling: A Review and an Empirical Analysis", In R.Slowinski and J. Weglarz, editors, Advances in project scheduling ,pages 113134.Elsevier,Amsterdam, Netherlands, 1989.

[8] F.F., Boctor, "Some Efficient Multi-heuristic Procedures for Resource-constrained Project Scheduling", European Journal of Operational Research, 49:3-13, 1990

[9] D.F., Cooper, "Heuristics for Scheduling Resource-constrained Projects: An Experimental Investigation" Management Science, 22:1186-1194, 1976.

[10] D.F., Cooper, "A Note on Serial and Parallel Heuristics for Resource-constrained Project Scheduling", Foundations of Control Engineering, 2:131-133, 1977.

[11] E.M., Davies, "An Experimental Investigation of Resource Allocation in Multi-activity Projects", Operations Research Quarterly, 24:587-591, 1973.

[12] E.W., Davis and J.H., Patterson, “ A Comparison of Heuristic and Optimum Solutions in Resource-constrained Project Scheduling”, Management Science , 21:944-955, 1975

[13] E.A., Elsayed, "Algorithms for Project Scheduling with Resource Constraints", International Journal of Production Research, 20:95-103, 1982

[14] R., Klein, "Bidirectional Planning: Improving Priority Rule Based Heuristics for Scheduling Resource -constrained Projects", European Journal of Operational Research, 127:619-638, 2000.

[15] R., Kolisch, "Project Scheduling under Resource Constraints-efficient Heuristics for Several Problem Classes", Physica, Hdeidelberg, 1995.

[16] R., Kolisch, "Efficient Priority Rules for the Resource-constrained Project Scheduling Problem", European Journal of Operations management, 14:179-192, 1996

[17] R., Kolisch, "Serial and Parallel Resourceconstrained Project Scheduling Methods Revisited: Theory and Computations Management", European Journal of Operational Research, 90:320-333, 1996.

[18] R., Kolisch and A., Drexl, "Adaptive Search for Solving Hard Project Scheduling Problems", Naval Research Logistics, 43:23-40, 1996

[19] S.R., Lawrene, Resource Constrained Project Scheduling- A Computational Comparison of Heuristic Scheduling Techniques, Technical Report, Carnegie Mellon University, Pittsburgh, Pennsylvania, 1985.

[20] K.Y., Li and R.J., Willis, "An Iterative Scheduling Technique for Resource-constrained
Project Scheduling, European Journal of Operational Research, 56: 370-379, 1992

[21] L., Ozdamar and G., Ulusoy, "A Local Constraint Based Analysis Approach to Project Scheduling Under General Resource Constraints", European Journal of Operational Research, 79: 287298, 1994.

[22] L., Ozdamar and G., Ulusoy, "An Iterative Local Constraint Based Analysis for Solving the Resource-constrained Project Scheduling Problem", European Journal of Operations management, 14: 193-208, 1996.

[23] L., Ozdamar and G., Ulusoy, "A Note on an Iterative Forward/Backward Scheduling Technique with Reference to a Procedure", by $\mathrm{Li}$ and Willis. European Journal of Operational Research, 89: 400407, 1996

[24] J.H., Patterson, "Alternate Methods of Project Scheduling with Limited Resources", Naval Research Logistics Quarterly, 20:767-784, 1973

[25] J.H., Patterson, "Project Scheduling: The Effects of Problem Structure on Heuristic Performance", Naval Research Logistics Quarterly, 23: 95-123, 1976

[26] A., Schrimer, "Case-based Reasoning and Improved Adaptive Search for Project Scheduling “, .Naval Research Logistics, 47: 201-222, 2000

[27] A., Schrimer, and S., Riesenberg, "Parameterized Heuristics for Project Schedulingbiased Random Sampling Methods," Manuskripte aus den instituten fur betriebswirtschaftslehre, 456, universitat kiel, germany, 1997.

[28] A., Schrimer and S., Riesenberg, "Class-based Control Schemes for Parameterized Project Scheduling Heuristics", Manuskripte aus den instituten fur betriebswirtschaftslehre 471, universitat kiel, germany, 1998.

[29] A., Thesen, "Heuristic Scheduling of Activities Under Resource and Precedence Restrictions", Management Science, 23: 412-422, 1976.

[30] P., R.Thomas, and S., Salhi, "An Investigation into the Relationship of Heuristic Performance with Network-resource Characteristics", Journal of Operational Research Society, 48: 34-43, 1997.

[31] G., Uiusoy, and L., Ozdamar, "Heuristic Performance and Network/Resource Characteristics in Resource-constrained Project Scheduling", Journal of Operational Research Society,40: 1145$1152,1989$.

[32] V., Valls,, M.A., Perez, and M.S., Quintanilla, "Heuristic Performance in Large Resourceconstrained Projects", Technical Report 92-2, Department of Statistics and Operations Research, University of Vaencia, 1992.

[33] G.E., Whitehouse, and J.R., Brown, "GENRES: An Extension of Brooks Algorithm for Project 
Scheduling with Resource Constraints", Computers \& Industrial Engineering, 3: 261-268, 1979.

[34] A., Lova, P., Tormos, and F., Barbe, "MultiMode Resource Constrained Project Scheduling: Scheduling Schemes, Priority Rules and Mode Selection Rules", Intelligence Artificial, V. 10, $\mathrm{N}^{\circ}$ 30, 2006.

[35] B., Paweł, E., M., Marek, S., Nski, and L., Podlodowski, "Novel heuristic solutions for Multi Skill Resource Constrained Project Scheduling Problem", Proceedings of the 2013 Federated Conference on Computer Science and Information Systems pp. 159-166

[36] S., Colak, A., Agrwal, and S.,Erenguc, “ MultiMode Resource-Constrained Project Scheduling Problem With Renewable Resources: New Solution Approaches", Journal of Business \& Economics Research - November 2013 Volume 11, Number 11.

[37] R., M., Chen, and S., T., LO, "Using an Enhanced Ant Colony System to Solve ResourceConstrained Project Scheduling Problem", IJCSNS International Journal of Computer Science and Network Security, VOL.6 No.11, November 2006

[38] M., Bastani, and S., H., Yakhchali, "Multi-mode Resource-Constraint Project Scheduling Problem (MRCPSP) with pre-emptive activities", nd International Conference on Mechanical, Automobile and Robotics Engineering (ICMAR'2013) March 1718, 2013 Dubai (UAE).

[39] A., Anderica, and C., Chira, "Best-Order Crossover in an Evolutionary Approach to MultiMode Resource-Constrained Project Scheduling", International Journal of Computer Information Systems and Industrial Management Applications. ISSN 2150-7988 Volume 6 (2014) pp., 364 - 372.
[40] J., Buddhakulsomsiri, and D., Kim, "Priority rule-based heuristic for multi-mode resourceconstrained project scheduling problems with resource vacations and activity splitting”, European Journal of Operational Research Volume 178, Issue 2, 16 April 2007, Pages 374-390.

[41] T., Browining, and A., A., Yassine, "Resourceconstrained multi-project scheduling: Priority rule performance revisited", International Journal of Production Economics Volume 126, Issue 2, August 2010, Pages 212-228.

[42] L., He, and L. Zhang, "Dynamic priority rulebased forward-backward heuristic algorithm for resource levelling problem in construction project", Journal of the Operational Research Society (2013), Volume: 64, Pages: $1106-1117$.

[43] A., Singh," Resource Constrained Multi-Project Scheduling with Priority Rules \& Analytic Hierarchy Process", Journal of the Operational Research Society, Procedia Engineering 69 (2014) 725 - 734, Elsevier Ltd.

[44] A., Lova, and P., Tormos, "Analysis of Scheduling Schemes and Heuristic Rules Performance in Resource-Constrained Multiproject Scheduling", Annals of Operations Research, February 2001, Volume 102, Issue 1-4, pp 263-286

[45] T., R., Browining, and A., A."Resource constrained multi-projects Scheduling: Priority Performance Revisited", Int. J., Production Economics, 2014.

[46] A. Atef, M. Abdel-Baset and I. Elhenawy," Composite Heuristic Priority Rules -Based on TieBreakers for Scheduling Multiple-Constrained Resource Projects", under publishing. 